\title{
Supported Liquid Membrane Using Hybrid Polyethersulfone/ Graphene Flat Sheet Membrane for Acetic Acid Removal
}

\author{
Norlisa Harruddin, ${ }^{1}$ Syed M. Saufi, ${ }^{1 *}$ Che Ku M. Faizal, ${ }^{2}$ Abdul Wahab Mohammad ${ }^{3}$ \\ and Huang Nay Ming 4 \\ ${ }^{1}$ Faculty of Chemical and Natural Resources Engineering, Universiti Malaysia Pahang, \\ Lebuhraya Tun Razak, 26300 Gambang, Pahang, Malaysia \\ ${ }^{2}$ Faculty of Engineering Technology, Universiti Malaysia Pahang, \\ Lebuhraya Tun Razak, 26300 Gambang, Pahang, Malaysia \\ ${ }^{3}$ Department of Chemical and Process Engineering, Faculty of Engineering and \\ Built Environment, Universiti Kebangsaan Malaysia, 43600 Bangi, Selangor, Malaysia \\ ${ }^{4}$ Department of Physics, Faculty of Science, University of Malaya, \\ 50603 Kuala Lumpur, Malaysia \\ ${ }^{*}$ Corresponding author: smsaufi@ump.edu.my
}

Published online: 15 February 2017

To cite this article: Harruddin, N. et al. (2017). Supported liquid membrane using hybrid polyethersulfone/graphene flat sheet membrane for acetic acid removal. J. Phys. Sci., 28(Supp. 1), 111-120, https://doi.org/10.21315/jps2017.28.s1.7

To link to this article: https://doi.org/10.21315/jps2017.28.s1.7

\begin{abstract}
Acetic acid is one of the inhibitors mainly found in biomass hydrolysates after acid hydrolysis of lignocellulose biomass. The presence of this compound can affect the final yield of bioethanol. Supported liquid membrane (SLM) is an efficient method for solute extraction from aqueous solution. However, unsuitable membrane structure and insufficient membrane strength are the typical problems experienced by the membrane support in the SLM system. In the current study, graphene nanopowder was incorporated into polyethersulfone (PES) dope polymer solution to fabricate flat sheet PES membrane support. The hydrophobicity of the PES membrane was increased from $85^{\circ}$ to $120^{\circ}$ when the graphene was added. The force required to break the PES/graphene membrane was 4.3N. This force was higher compared to the pristine PES membrane which was 1.9N. Both membranes however showed almost similar acetic acid removal percentages which were $86 \%$ and $83 \%$ for pristine PES and PES/graphene membrane, respectively.
\end{abstract}

Keywords: Acetic acid, liquid membrane, hybrid Polyethersulfone, graphene, flat sheet membrane 


\section{INTRODUCTION}

Meeting the world's demand on fuels and value-added chemicals from many sectors requires resources that are abundant and inexpensive to produce. Lignocellulosic biomass has been pointed out to be one of the most promising alternative sources. It is the only sustainable source of organic carbon on earth for the production of fuels and fine chemicals with net zero carbon emission. To make it reusable, lignocellulosic biomass has to undergo pretreatment and hydrolysis processes to release the sugar component for further processing to the final targeted products. However, these treatment processes induce the formation of inhibitor which directly restricts the microorganism activity during the fermentation process. Acetic acid is found to be the most serious and abundant inhibitor present in lignocellulosic biomass hydrolysate. Therefore, it is crucial to remove acetic acid to avoid inhibition problems affecting microorganisms prior to fermentation.

Liquid membrane process for solute extraction has gained increasing attention for its high selectivity and separation factors. ${ }^{1}$ The extraction and stripping occur simultaneously in liquid membrane, hence maximum separation can be achieved. Liquid membrane system consists of three phases which are feed, strip and organic liquid membrane phases. During extraction steps, the carrier inside the liquid membrane phase extracts the solute from the feed phase. The extracted solute was then stripped by using a stripping agent in the strip phase. The carrier will remain in the organic liquid membrane phase to extract the remaining solute from the feed phase.

The organic liquid membrane is normally immobilised in the microporous membrane support known as supported liquid membrane (SLM). The application of SLM in industrial is still limited due to the insufficient stability of the SLM system. Loss of carrier or solvent from the membrane support, blockage of membrane pores with carrier, and progressive wetting of the membrane pores by aqueous phase have been identified as the main challenges that need to be addressed in the SLM process. The ideal membrane support for SLM should have high tensile strength, high hydrophobicity, and highly resistant to chemical.

Several methods can be used to improve the physical characteristic of membrane support. One of the simple methods used to alter the properties of the membrane support is by adding an inorganic compound into the polymer dope solution. ${ }^{2}$ The most commonly applied inorganic materials include carbon molecular sieves, zeolites, metal organic frameworks, carbon nanotubes and graphene-based 
materials. Due to the unique characteristic of the graphene, various studies have been focussed on the development of hybrid membrane based on different graphene derivatives recently.

$\mathrm{Wu}$ et al.had incorporated graphene nanosheet in a polyvinylpyrolidone membrane in order to improve membrane hydrophobicity for $\mathrm{CO}_{2}$ absoprtion. ${ }^{2}$ They found out that other than hydrophobicity, the addition of $1 \%$ graphene improved the mechanical strength of the hybrid membrane significantly. However, when the concentration of graphene was increased from 3\% to $7 \%$, no significant change on the mechanical strength was observed. Park et al. had synthesised thin film composite membrane by incorporating graphene oxide nanosheet in polysulfone solution. ${ }^{3}$ The hybrid graphene oxide nanosheet membrane showed water permeability improvement due to the enhancement of the membrane hydrophilicity. Dong et al. blended reduced graphene oxide with Pebax 1657 polymer to prepare mixed matrix membrane for separation of $\mathrm{CO}_{2} / \mathrm{N}_{2} \cdot{ }^{4}$ Based on their results, the permeability of $\mathrm{CO}_{2}$ improved and selectivity of $\mathrm{CO}_{2} / \mathrm{N}_{2}$ were increased.

Based on the previous studies, graphene contains extraordinary electronic, thermal, and mechanical properties. In this study, graphene was selected as inorganic filler in order to improve the hydrophobicity and mechanical strength of the membrane support in the SLM system. Graphene was incorporated into PES dope polymer solution and flat sheet membranes were prepared using vapour induced phase separation (VIPS) technique. The strength and hydrophobicity of the membrane was compared and the performance for acetic acid removal using the SLM system was evaluated.

\section{EXPERIMENTAL}

\subsection{Materials}

Polyethersulfone (Radel ${ }^{\circledR}$ A, Solvay, USA) was used as the base polymer for membrane fabrication. The polymer was dried for $24 \mathrm{~h}$ at $60^{\circ} \mathrm{C}$. Dimethylacetamide (DMaC) and polyethylene glycol (PEG 200) were purchased from Sigma Aldrich and were used as solvent and additive, respectively. Distilled water was used as a coagulation medium. The graphene nanopowder used as an inorganic compound was kindly supplied by Low Dimensional Materials Research Centre, University of Malaya, Malaysia.

In SLM system, acetic acid (Sigma) solution was used as a feed phase. For organic liquid membrane phase preparation, tri-n-octylamine (TOA) and 2-ethyl-1-hexanol 
was used as carrier and diluent, respectively. Both chemicals were supplied by Sigma Aldrich. Sodium hydroxide (Mercks) was used as a stripping agent.

\subsection{Membrane Fabrication}

The polymeric membrane support was fabricated by using vapour inducing phase separation technique. Two types of dope solution were prepared as shown in Table 1, with graphene and without graphene addition. For graphene-based dope solution, graphene nanopowder was dissolved in $\mathrm{DMaC}$ solvent and ultrasonic for almost 1 day. Then the PEG and PES were dissolved into this premixed DMaC solvent under continuous stirring up to $48 \mathrm{~h}$ at room temperature until homogenous dope solution was formed. The dope polymer solution was degassed at room temperature for $24 \mathrm{~h}$. Flat sheet membrane was casted using semi-automatic casting machine onto glass plate and exposed to air environment at $86 \%$ relative humidity for $30 \mathrm{~s}$. The cast film was then immersed into water coagulation bath at temperature of $40^{\circ} \mathrm{C}$ to induce complete solidification process. 30 min later, the solidified film was transferred to another water coagulation bath for 1 day and dried at room temperature for 2 days.

Table 1: Dope polymer composition.

\begin{tabular}{ll}
\hline Membrane & Composition \\
\hline PES & $15 \%$ PES, 42.5\% PEG 200, 42.5\% DMaC, \\
PES/Graphene & $15 \%$ PSF, 42.5\% PEG 200, 42.5\% DMaC, 0.1wt \% graphene \\
\hline
\end{tabular}

\subsection{Supported Liquid Membrane System}

The membrane support $(10.5 \mathrm{~cm} \times 4 \mathrm{~cm})$ was incubated in organic liquid membrane solution of $0.5 \mathrm{M}$ TOA in 2-ethyl-1-hexanol for almost $24 \mathrm{~h}$. Filter paper was used to remove the excess organic liquid membrane on the membrane surface. The supported membrane was placed in membrane cell and attached to the SLM system as shown in Figure 1. The membrane cell was made of two Teflon compartments of equal size with the dimension of $16.5 \mathrm{~cm} \times 10 \mathrm{~cm} .10 \mathrm{~g} / \mathrm{L}$ acetic acid solution and $0.5 \mathrm{M} \mathrm{NaOH}$ solution were used as the feed and stripping solution, respectively. The solutions were circulated into the membrane cell by two-channel peristaltic pump. The concentration acetic acid was detected by Synergy Hydro C18 HPLC column (Phenomenex, $150 \mathrm{~mm} \times 4.6 \mathrm{~mm}, 4 \mu \mathrm{m}$ particle size) connected to Waters Acquity UPLC system. $0.02 \mathrm{M}$ potassium dihydrogen phosphate was used as mobile phase and acetic acid was detected by UV detector at $221 \mathrm{~nm}$ wavelength. 


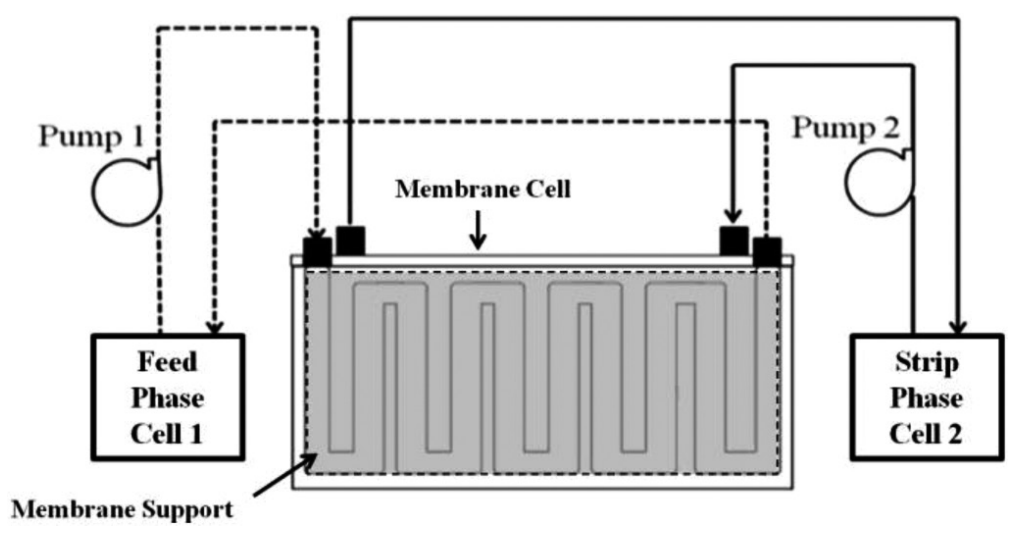

Figure 1: Schematic diagram of supported liquid membrane system.

\subsection{Acetic Acid Removal Percentage}

Equation 1 was used to calculate the percentage of acetic acid removal during the SLM process:

$$
\operatorname{Removal}(\%)=\frac{[\mathrm{AA}]_{\mathrm{fi}}-[\mathrm{AA}]_{\mathrm{fo}}}{[\mathrm{AA}]_{\mathrm{fi}}} \times 100
$$

where

$[\mathrm{AA}]_{\mathrm{fi}}$ is the initial concentration of acetic acid ions in the feed phase, and $[\mathrm{AA}]_{\mathrm{fo}}$ is the final concentration of acetic acid ions in feed phase.

\subsection{Membrane Characterisation}

The membrane morphology was observed by using scanning electron microscope (SEM) ZEISS EVO 50. The membrane was fractured in liquid nitrogen and coated with gold-palladium before examined in SEM machine. Optical contact angle measurement system (CAM 101 optical Contact Angle Meter, KSV Instruments) was used to determine the surface hydrophobicity of the membranes at room temperature. At least three measurements were performed at different membrane locations to obtain the average contact angle for one membrane sample. The mechanical properties of the membrane support were tested using Tensile Test Shimadzu EZ-LX. 


\section{RESULTS AND DISCUSSION}

\subsection{Morphology of Membrane Support}

Membrane morphology such as pore structure and size plays a crucial role to the performance of SLM process since the liquid membrane is held within the pores of the membrane by capillary force. The liquid membrane must remain stable in order to stay inside the pores for a long duration of time. The morphology of the pristine PES and PES/graphene hybrid membrane were shown in Figure 2. Both membranes had almost symmetric structure. A symmetric membrane is suitable for the SLM process due to the amount of forces exerted on both sides of the membranes are identical, thus the membrane stability improved during the SLM process. ${ }^{5,6}$ It can be seen on Figure 2(a) that pristine PES membrane showed a symmetric structure with cylindrical microvoids uniformly distributed throughout the cross section of membrane. Addition of graphene induced the dense sublayer on the bottom of the membrane and the distance between two cylindrical microvoids was increased.

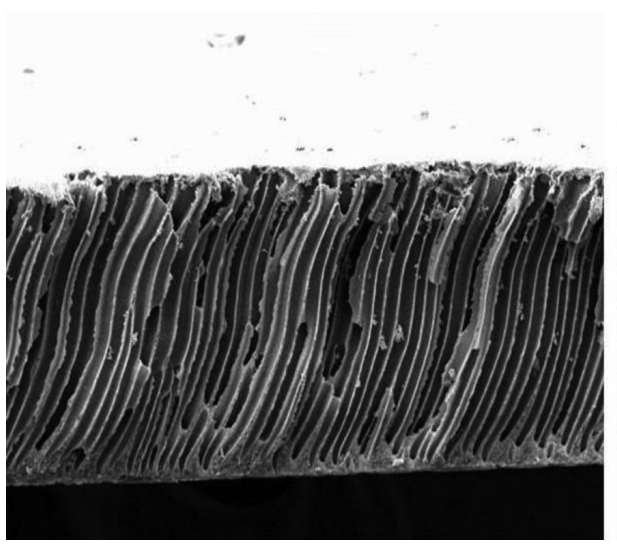

(a)

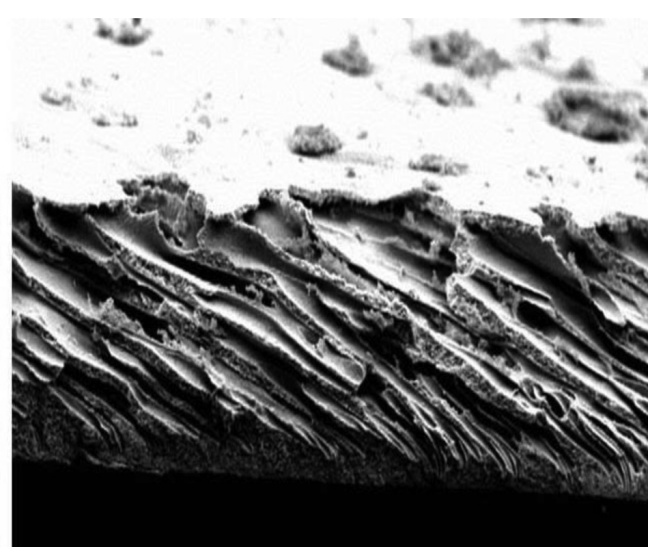

(b)

Figure 2: Cross section of membrane support, Mag: $300 \mathrm{~K} \times$ for (a) Pristine PES, and (b) PES/graphene hybrid membrane.

\subsection{Contact Angle}

Figure 3 exhibits the contact angle of: (a) pristine PES membrane; and (b) PES/ graphene hybrid membrane. The contact angle of PES/graphene membrane increased up to $41 \%$ compared to the pristine PES membrane. Pristine PES membrane almost achieving hydrophobic material $\left(<90^{\circ}\right)$, but with graphene addition it transformed to nearly superhydrophobic material $\left(<150^{\circ}\right)$. It is 
found that graphene concentration as low as $0.1 \%$ managed to increase the hydrophobicity of the membrane support significantly. The increment of membrane support hydrophobicity will enhance the stability of the organic liquid membrane phase within the membrane pore by preventing liquid membrane leakage.

Contact Angle: $85^{\circ}$

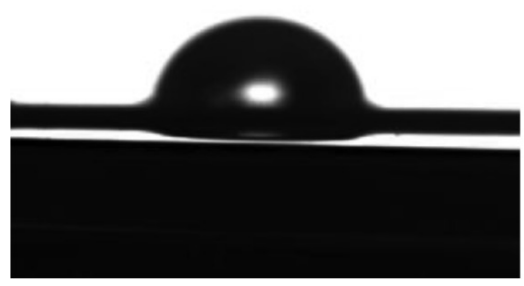

(a)
Contact Angle: $120^{\circ}$

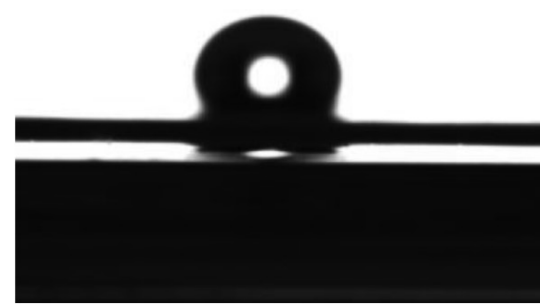

(b)

Figure 3: Contact angle of (a) pristine PES membrane, and (b) PES/graphene hybrid membrane.

\subsection{Tensile Strength}

Figure 4 shows the force required to break the membrane sample during mechanical testing. The pristine PES membrane easily broke with $1.9 \mathrm{~N}$ force. The elongation before breakage of the membrane increased drastically when added with graphene. The required force to break the PES/graphene membrane is $4.3 \mathrm{~N}$ which is about $126 \%$ higher than pristine PES membrane. When nanofillers are properly dispersed in a host polymer, they can spread the load transfer of the composite material, thereby improving its mechanical properties. ${ }^{7}$ Graphene particles also can influence the PES crystallinity and somehow affect the mechanical strength of membrane. ${ }^{8}$ Although high pressure is not applied during SLM process, good mechanical properties of the membrane support can improve the lifetime of the membrane in SLM process.

In SLM process, the membrane will have the tendency to suffer breakage problem after long duration of experiment. Membrane exhibits lower mechanical strength and tends to crack, which results in a loss of membrane performance. The phenomenon of membrane breakage is not surprising because previous studies have revealed that membranes with low tensile strength and integrity structure often face a fouling and breakage problem. ${ }^{9}{ }^{10}$ Hence, the membrane must have high tensile strength so that it remains stable without any breakage after long duration of time. 


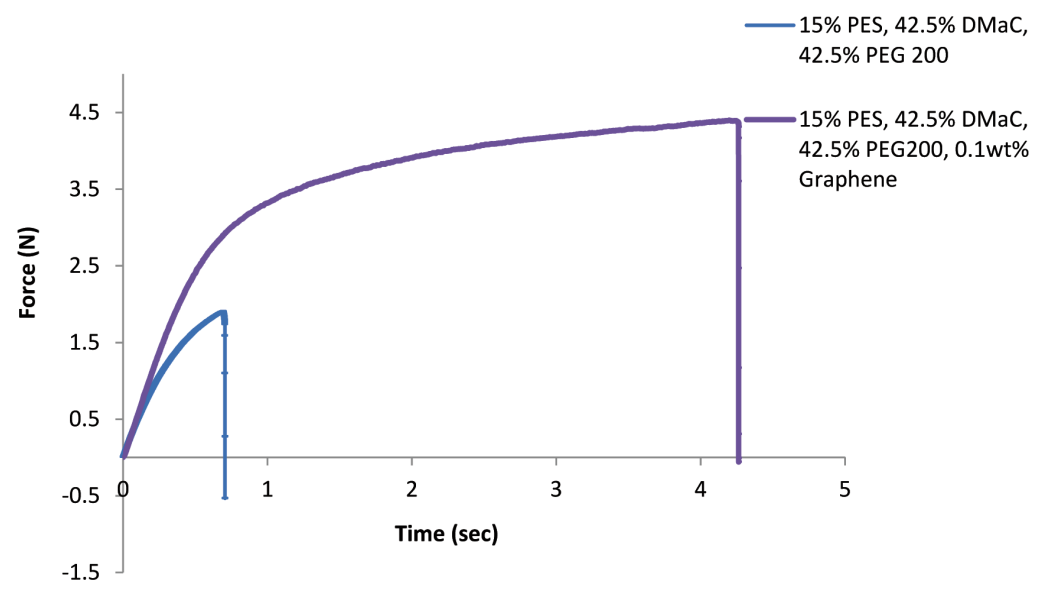

Figure 4: Plot of stress vs. time for pristine PES membrane and PES/graphene membrane.

\subsection{SLM Performance for Acetic Acid Removal}

The removal percentage of acetic acid from the aqueous phase using pristine PES and PES/graphene hybrid membrane is shown in Figure 5. The acetic acid removal percentage increases with the processing time. However, extraction time was fixed to $8 \mathrm{~h}$ during this study in order to achieve high removal percentage at short extraction time. Pristine PES and PES/graphene membrane were able to remove $86 \%$ and $83 \%$ of acetic acid, respectively. Both membranes remain stable within $8 \mathrm{~h}$ of SLM process. No obvious effect on the acetic acid performance was

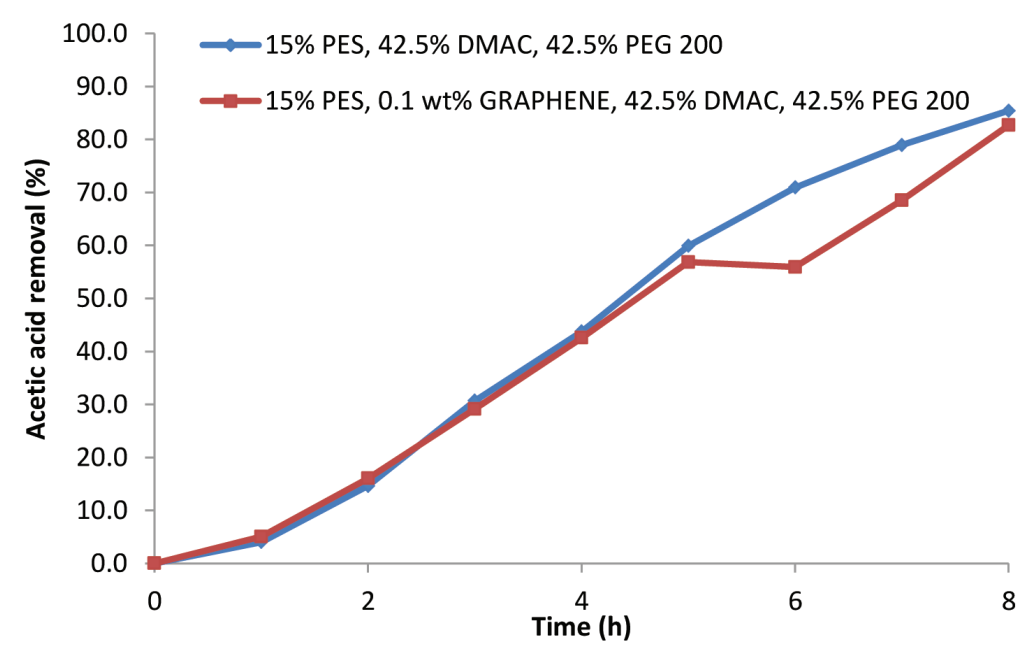

Figure 5: Schematic diagram of supported liquid membrane system. 
observed although both membranes had different hydrophobicity and mechanical strength. The hydrophobicity of the pristine PES membrane $\left(85^{\circ}\right)$ is already more than enough to contain the organic liquid membrane phase in the membrane pore. However, we expected that the effect of graphene in the SLM process become more important if the membrane was applied in the real biomass hydrolysate solution in the future experiment.

\section{CONCLUSION}

Addition of graphene as low as $0.1 \mathrm{wt} . \%$ in PES dope polymer solution enhanced the membrane hydrophobicity and mechanical properties. However, the acetic acid removal performance for both membranes is almost the same and not affected by membrane hydrophobicity and mechanical strength.

\section{ACKNOWLEDGEMENTS}

The authors were grateful for the research financial support by the Ministry of Higher Education Malaysia under the Fundamental Research Grant Scheme (FRGS RDU140144), the Long-term Research Grant Scheme (LRGS-UKM on Future Biorefineries), and Universiti Malaysia Pahang under the Research University Grant Scheme (RDU140337). Norlisa Harruddin is grateful for the sponsorship given by the Ministry of Higher Education under MyBrain15 (MyPhD) scheme and Universiti Malaysia Pahang under Graduate Research Scheme (GRS 150303) throughout her doctoral study.

\section{REFFERENCES}

1. Efome, J. E. et al. (2015). Effects of superhydrophobic $\mathrm{SiO}_{2}$ nanoparticles on the performance of PVDF flat sheet membranes for vacuum membrane distillation. Desalin., 373, 47-57, https://doi.org/10.1016/j. desal.2015.07.002.

2. $\mathrm{Wu}, \mathrm{X}$. et al. (2016). Hydrophobic PVDF / graphene hybrid membrane for $\mathrm{CO}_{2}$ absorption in membrane contactor. J. Memb. Sci., 520, 120-129, http://dx.doi.org/10.1016\%2Fj.memsci.2016.07.025.

3. Park, M. J. et al. (2015). Graphene oxide incorporated polysulfone substrate for the fabrication of flat-sheet thin-film composite forward osmosis membranes. J. Memb. Sci., 493 (2015) 496-507, http://dx.doi. org/10.1016\%2Fj.memsci.2015.06.053. 
4. Dong, G. et al. (2016). Enhanced $\mathrm{CO}_{2} / \mathrm{N}_{2}$ separation by porous reduced graphene oxide/Pebax mixed matrix membranes, J. Memb. Sci., 520, 860868, https://doi.org/10.1016/j.memsci.2016.08.059.

5. Scott, K. \& Hughes, R. (1996). Industrial membrane separation technology. Ireland: Springer.

6. Lv, J. et al. (2007). Exploration of heavy metal ions transmembrane flux enhancement across a supported liquid membrane by appropriate carrier selection. Chem. Eng. Sci., 62(21), 6032-6039.

7. Chul, Y. et al. (2016). Water desalination using graphene-enhanced electrospun nano fiber membrane via air gap membrane distillation. J. Memb. Sci., 520,99-110, https://doi.org/10.1016/j.memsci.2016.07.049.

8. Moradi, R. et al. (2015). Preparation and characterization of polyvinylidene fluoride/graphene superhydrophobic fibrous films. Polym., 7, 1444-1463, http://dx.doi.org/10.3390/polym7081444.

9. Khayet, M. \& Matsuura, T. (2011). Membrane distillation: Principles and applications. Amsterdam: Elsevier.

10. Lin, Y. K. et al. (2009). Formation of isotactic polypropylene membranes with bicontinuous structure and good strength via thermally induced phase separation method. Desalin., 236(1-3), 8-15, https://doi.org/10.1016/j. desal.2007.10.044. 